\title{
Effect of lower limb preference on variability of centre of pressure movement during gait
}

\author{
Zdeněk Svoboda ${ }^{1, *}$, Lucia Bizovská1 ${ }^{1}$ Zuzana Kováčiková ${ }^{1}$, Patrik Kutílek ${ }^{2}$, Roman Cuberek ${ }^{1}$, \\ and Miroslav Janura ${ }^{1}$
}

\author{
${ }^{1}$ Faculty of Physical Culture, Palacký University Olomouc, Olomouc, Czech Republic; and ${ }^{2}$ Faculty of Biomedical Engineer- \\ ing, Czech Technical University in Prague, Kladno, Czech Republic
}

Copyright: (c) 2015 Z. Svoboda et al. This is an open access article licensed under the Creative Commons Attribution License (http://creativecommons.org/licenses/by/4.0/).

\begin{abstract}
Background: Centre of pressure (COP) movement variability, as an example of 'end-point' variability (stability), may be one of the indicators that can assess the functional gait asymmetry caused by foot preference in relation to fall risk. Objective: The aim of this study was to investigate the possible differences in COP movement variability during the stance phase of gait caused by both aging and lower limb preference. Methods: Two groups of females participated in this study: Younger ( $n=25$, age $22.2 \pm 1.8$ years) and Middle-aged ( $n=25$, age $56.6 \pm 4.9$ years). COP movement and ground reaction forces during gait at self-selected speed were recorded using two force platforms. The standard deviations of the medial-lateral and anterior-posterior COP displacements in four subphases: loading response (LR), midstance (MSt), terminal stance (TSt) and pre-swing (PSw) were assessed. Results: The observed variables indicated significantly higher mean values in almost all cases in subphases LR and PSw in comparison with MSt and TSt. When comparing preferred and non-preferred limb, the preferred limb showed greater variability in medial-lateral direction during LR and less variability in anterior-posterior direction during PSw. In the Younger group, greater variability was found on preferred limb in anterior-posterior direction during LR. When assessing age-related differences all significant cases showed higher variability in the Middle-aged group. Conclusions: The results suggest that COP movement variability is less on the non-preferred limb during weight acceptance and on the preferred limb during propulsion.
\end{abstract}

Keywords: fall risk, ground reaction force, limb dominance, limb preference, stability, walking

\section{Introduction}

Variability in human walking arises from many potential sources, including natural temporal dynamics of neuromotor control (da Costa, Batistão, \& Rocha, 2013), pathologies of the neurological or musculoskeletal systems (Kaipust, Huisinga, Filipi, \& Stergiou, 2012; Webster, Merory, \& Wittwer, 2006), the effects of aging (Barak, Wagenaar, \& Holt, 2006; Callisaya, Blizzard, Schmidt, McGinley, \& Srikanth, 2010), shoe type (Jandová, Froňková, \& Nováková, 2013) as well as variations in external environment (Chau, Young, \& Redekop, 2005).

\footnotetext{
* Address for correspondence: Zdeněk Svoboda, Department of Natural Sciences in Kinanthropology, Faculty of Physical Culture, Palacký University Olomouc, třída Míru 117, 77111 Olomouc, Czech Republic. E-mail: zdenek.svoboda@upol.cz
}

We can consider two basic concepts of variability: the coordinative variability (proximal joints of the body) and 'end-point' variability (distal segments of the body) (Hamill, Palmer, \& Van Emmerik, 2012). From this point of view we can suppose greater coordinative variability and less 'end-point' variability in healthy young subjects in comparison with older subjects or subjects with some disability.

Similarly supposition would be stated for limb preference. Some authors (Peters, 1988) recognize manipulative or mobilizing (preferred) limb and limb used for support of an action (stabilising, non-preferred). From this point of view in the manipulative limb coordinative variability would be expected greater and 'end-point' variability less, while in the stabilizing limb coordinative variability less and 'end-point' variability greater (Peters, 1988).

Centre of pressure (COP) movement variability, as an example of 'end-point' variability (stability), may be 
one of the indicators that can assess the role of the preferred and non-preferred limbs, and age in stabilization during gait. Although the relationship between COP variability during gait and balance or fall risk has not been studied in the scientific literature yet, it could be a potential variable for dynamic balance assessment. It was showed that in subjects with fall history, gait variability in temporal-spatial variables such as stride length (Reelick et al., 2011; Taylor, Delbaere, Mikolaizak, Lord, \& Close, 2013), stride time (Hausdorff, Edelberg, Mitchell, Goldberger, \& Wie, 1997; Miyoshi, Kinugasa, Urushihata, \& Soma, 2011), swing time (Taylor et al., 2013) or minimum foot clearance (Barrett, Mills, \& Begg, 2010) and kinematic variables (Barak et al., 2006) is increased.

In the scientific literature we did not find any study concerning these consideration thus we hypothesized that COP movement variability would be greater in older subjects (less stability) and would differ in preferred and non-preferred limbs.

The aim of this study was to identify differences in COP movement variability between preferred and nonpreferred lower limbs and to investigate age-related differences in COP movement variability independently for preferred and non-preferred limbs in various gait cycle phases.

\section{Materials and methods}

\section{Subjects and experimental setup}

The participants in this study were two groups of healthy females. Their physical characteristics are shown in Table 1. All participants did not have any musculoskeletal or neurological problems at the time of measurement and at least half year before. Participants in the Younger group $(n=25)$ were students at a local university, which perform sport only on a recreational level and subjects in the Middle-aged group $(n=25)$ were chosen as individuals with various sedentary jobs who were in moderate physical condition.

Participants were asked to walk barefoot along an $8 \mathrm{~m}$ walkway at a self-selected speed (their common walking

Table 1

Participants characterisation (mean $\pm S D$ )

\begin{tabular}{lcc}
\hline & $\begin{array}{c}\text { Younger } \\
\text { women }(n=25)\end{array}$ & $\begin{array}{c}\text { Middle-aged } \\
\text { women }(n=25)\end{array}$ \\
\hline Age (years) & $22.16 \pm 1.80$ & $56.63 \pm 4.85$ \\
Weight $(\mathrm{kg})$ & $61.24 \pm 6.99$ & $70.53 \pm 13.92$ \\
Height $(\mathrm{m})$ & $1.68 \pm 0.06$ & $1.62 \pm 0.06$ \\
BMI $\left(\mathrm{kg} \cdot \mathrm{m}^{-2}\right)$ & $21.74 \pm 1.61$ & $26.71 \pm 5.02$ \\
\hline
\end{tabular}

speed) and to perform 8-10 trials. The mean walking speed was similar in the groups, $1.22( \pm 0.11) \mathrm{m} \cdot \mathrm{s}^{-1}$ in the Middle-aged group and $1.23( \pm 0.06) \mathrm{m} \cdot \mathrm{s}^{-1}$ in the Younger group, so possible effects of speed were not considered. The first three trials were excluded from further analysis for familiarisation with the experimental environment. Two force plates (Kistler 9286AA, Kistler Instrumente, Winterthur, Switzerland) placed sequentially in the middle of the walkway were used to record ground reaction force values and COP movement. Sampling frequency was set at $200 \mathrm{~Hz}$ as natural frequency of the force plates. To avoid the influence of initiation and termination of gait, only gait cycles in middle of the walkway were recorded. The preferred limb was determined before the start of the measurement by asking a question: "Which leg would you kick a ball with?". The limb which participants answered the question with was considered as preferred.

The study was approved by the institutional research ethics committee and the participants provided written informed consent.

\section{Data processing}

Stance phase was determined using vertical ground reaction force (vGRF) values with a minimum of 5\% of body weight. Data were filtered using a third-order low-pass Butterworth filter with a cut-off frequency of $30 \mathrm{~Hz}$, using MATLAB software (MATLAB R2010b; MathWorks, Natick, MA, USA).

Based on vGRF behaviour, stance phase for each foot was divided into four subphases, as described by Ayyappa (1997). Subphases were identified as follows: loading response (LR) - time interval between heel strike and first peak of vGRF, midstance (MSt) - time interval from the first peak of vGRF to minimum of vGRF in the middle of the stance phase, terminal stance (TSt) - time interval between minimum of vGRF in the middle of the stance phase and the second peak of VGRF, pre-swing (PSw) - time interval between second peak of vGRF and toe off.

Instantaneous displacements in the medial-lateral (Dx) and anterior-posterior (Dy) directions in each time interval $(0.005 \mathrm{~s})$ were defined as absolute values of the differences between two consecutive recorded COP positions in each direction.

Standard deviations of variables in each subphase defined above were used in further analysis as variability indicators. All calculations were performed using custom-written MATLAB programs (MATLAB R2010b, MathWorks, Natick, MA, USA) separately for the preferred and non-preferred limb. 


\section{Statistical analysis}

Means of five trials from each subject were used for further analysis. Statistical analysis was performed using Statistica (Version 12.0; StatSoft, Tulsa, OK, USA). The Kolmogorov-Smirnov test was used for verification of normality. Multifactorial ANOVA and Tukey post-hoc test were used to determinate the differences between preferred and non-preferred limbs within both groups, age-related differences for both limbs and differences between various gait cycle phases for both limbs and groups. The significance level was set at $p=.05$. For variables evidencing a statistical difference, the effect size was evaluated using Cohen's $d$, and only values higher than 0.8 (evidencing a large effect) were deemed significant.

\section{Results}

Means and standard deviations of variability indicators (for description in more detail see methods section) in preferred and non-preferred limb are summarised in Table 2. In all significant differences a large effect size was found.

When comparing preferred and non-preferred limb within the Middle-aged group, the preferred limb showed greater variability in medial-lateral direction during LR and less variability in anterior-posterior direction during subphase PSw. In the Younger group differences between limbs were similar as in the Middleaged group but only the difference in anterior-posterior direction during LR (variability on preferred limb was greater) was significant.

When assessing age-related differences all significant cases showed higher variability of COP in Middleaged group. Most of the significant differences were found in subphase PSw.

Variability of COP movement was significantly greater $(p<.05)$ during LR (in all cases) and PSw (in all cases with exception of preferred limb in Younger group) in comparison with MSt and TSt. There was no significant difference in COP movement variability between MSt and TSt phases. Variability during PSw in comparison with LR was significantly less in all cases (both groups, both directions) for preferred limb, significantly less on non-preferred limb for medial-lateral direction in the Younger group and significantly greater on non-preferred limb for anterior-posterior direction in the Middle-aged group.

\section{Discussion}

This study proposed the assessment of COP variability during gait in various stance phase subphases using a procedure for stance phase division in regard to vGRF

Table 2

Observed gait variables for preferred and non-preferred limb in different subphases of a stance phase. All values are in millimetres presented as mean $\pm S D$.

\begin{tabular}{|c|c|c|c|c|}
\hline & \multicolumn{2}{|c|}{ Younger women } & \multicolumn{2}{|c|}{ Middle-aged women } \\
\hline & Preferred limb & Non-preferred limb & Preferred limb & Non-preferred limb \\
\hline \multicolumn{5}{|c|}{ Loading response } \\
\hline SD Dx & $2.08 \pm 1.05$ & $2.89 \pm 1.43$ & $5.26 \pm 2.21^{\#}$ & $3.32 \pm 1.47^{*}$ \\
\hline SD Dy & $7.02 \pm 3.34$ & $4.15 \pm 2.51^{*}$ & $6.61 \pm 3.27$ & $4.79 \pm 1.89$ \\
\hline \multicolumn{5}{|l|}{ Midstance } \\
\hline SD Dx & $0.18 \pm 0.04$ & $0.17 \pm 0.05$ & $0.16 \pm 0.06$ & $0.14 \pm 0.05$ \\
\hline SD Dy & $0.63 \pm 0.14$ & $0.66 \pm 0.14$ & $0.70 \pm 0.29$ & $0.65 \pm 0.27$ \\
\hline \multicolumn{5}{|c|}{ Terminal stance } \\
\hline SD Dx & $0.14 \pm 0.03$ & $0.15 \pm 0.03$ & $0.12 \pm 0.03$ & $0.13 \pm 0.04$ \\
\hline SD Dy & $0.55 \pm 0.21$ & $0.51 \pm 0.16$ & $0.62 \pm 0.27$ & $0.54 \pm 0.29$ \\
\hline \multicolumn{5}{|l|}{ Pre-swing } \\
\hline SD Dx & $0.84 \pm 0.34$ & $1.19 \pm 0.51$ & $1.89 \pm 0.68^{\#}$ & $2.54 \pm 1.84^{\&}$ \\
\hline SD Dy & $1.97 \pm 0.70$ & $3.16 \pm 1.23$ & $3.66 \pm 2.86$ & $8.93 \pm 7.45^{* \&}$ \\
\hline
\end{tabular}

Note. SD Dx (Dy) = standard deviation of COP displacement in medial-lateral (anterior-posterior) direction. ${ }^{*} p<.05$ when comparing preferred vs. non-preferred limb within groups, ${ }^{\#} p<.05$ when comparing preferred limb between groups, ${ }^{\&} p<.05$ when comparing non-preferred limb between groups. 
recorded by force platforms. Preferred and non-preferred limbs were considered separately.

\section{Variability of COP movement in various subphases of a stance phase}

The observed variables showed higher mean values in subphases LR and PSw than in MSt and TSt. MSt and TSt are parts of single support, which is characterised by maintaining limb and trunk stability (Perry $\&$ Burnfield, 2010). Thus movement variability can be considered an indirect assessment of stability (Wang \& Watanabe, 2012), lower variability of COP movement in these two subphases could indicate higher stability of gait. COP movement is specific in every subphase; therefore, more attention will be given to stability assessment in each separate subphase.

\section{Variability of COP movement in preferred and non-pre- ferred limbs}

When considering within-group differences between preferred and non-preferred limbs, COP movement variability showed significantly less value on non-preferred limb during LR for anterior-posterior direction in the Younger group and medial-lateral direction in the Middle-aged group. During PSw, COP movement variability showed greater value on the non-preferred limb (significantly only for anterior-posterior direction in Middle-aged group).

If limbs are considered as non-preferred for stability and preferred for mobility, then our results suggest association between their function and gait cycle subphase. During LR when lower limb control weight acceptance and remain stability, variability of COP movement would be expected less on stabilizing limb, during PSw when lower limb ensures propulsion and movement forward then mobility limb could show less COP movement variability. No significant differences between limbs were found in subphases MSt and TSt. These results suggest behaviour of lower limbs in subphases MSt and TSt was considered to be symmetrical.

Wang and Watanabe (2012) presented greater variability of COP movement in the mediolateral direction across whole stance phase in the non-preferred limb in comparison with preferred. In our study this tendency is uniform only for the Younger group.

\section{Age-related differences in COP variability}

When considering age-related differences in COP variability regardless limb, it increases with age (Bizovska et al., 2014). In the preferred limb, significantly higher variability of COP movement in medial-lateral direction was found in LR and PSw in the Middle-aged group. For the non-preferred limb, significantly greater variability was present only in subphase PSw in both medial-lateral and anterior-posterior directions in the Middle-age group. Therefore, it can be assumed that the function of the non-preferred limb as a stabilising agent during LR does not rapidly change with increasing age. For the preferred limb, age-related changes were found mainly in the double-support parts of a stance phase (subphases LR and PSw) in medial-lateral direction. This could indicate that with increasing age, medial-lateral stability is worsened. Similar findings for quiet stance confirmed (Abrahamova \& Hlavacka, 2008).

\section{Limitations}

Level of preference was not observed, and only one test was used for lower limb preference verification. Although assessment of limb preference with more tests would be beneficial, the ball-kick test was chosen because of its clear bilateral information. The COP movement can be influenced by foot type and other morphological factors that were not considered in this study.

\section{Conclusions}

This study showed differences in lower limb behaviour during gait by assessing the variability of COP movement between Younger and Middle-age groups and between preferred and non-preferred limbs. Significant differences were found only in subphases of loading response and pre-swing which are associated with weight acceptance and propulsion. These results also show, that COP movement variability increases with age. In the Middle-aged group less variability of COP movement was found on the non-preferred limb during weight acceptance (loading response phase) and on the preferred limb during propulsion (pre-swing phase). Future research assessing bilateral behaviour of lower limbs during gait in various age groups from children to elderly individuals is warranted.

\section{Acknowledgments}

The study was supported by a research grant from the Czech Science Foundation (No. 13-32105S) "Analysis of health-related benefits of walking: Assessment of walking intervention in sedentary adults" and by a grant from the European Social Fund and the Government of the Czech Republic (No. CZ.1.07/2.3.00/30.0004) "Support for the creation of excellent research teams and intersectoral mobility at Palacký University in Olomouc". 


\section{References}

Abrahamova, D., \& Hlavacka, F. (2008). Age-related changes of human balance during quiet stance. Physiological Research, 57, 957-964.

Ayyappa, E. (1997). Normal human locomotion, part 2: Motion, ground-reaction force and muscle activity. Journal of Prosthetics and Orthotics, 9, 49-57.

Barak, Y., Wagenaar, R. C., \& Holt, K. G. (2006). Gait characteristics of elderly people with a history of falls: A dynamic approach. Physical Therapy, 86, 1501-1510. doi:10.2522/ptj.20050387

Barrett, R. S., Mills, P. M., \& Begg, R. K. (2010). A systematic review of the effect of ageing and falls history on minimum foot clearance characteristics during level walking. Gait \& Posture, 32, 429-435. doi:10.1016/j.gaitpost.2010.07.010

Bizovska, L., Svoboda, Z., Kutilek, P., Janura, M., Gaba, A., \& Kovacikova, Z. (2014). Variability of centre of pressure movement during gait in young and middleaged women. Gait \& Posture, 40, 399-402. doi:10.1016/j. gaitpost.2014.05.065

Callisaya, M. L., Blizzard, L., Schmidt, M. D., McGinley, J. L., \& Srikanth, V. K. (2010). Ageing and gait variability: A population-based study of older people. Age and Ageing, 39, 191-197. doi:10.1093/ageing/afp250

Chau, T., Young, S., \& Redekop, S. (2005). Managing variability in the summary and comparison of gait data. Journal of NeuroEngineering and Rehabilitation, 2, 22.

da Costa, C. S., Batistao, M. V., \& Rocha, N. A. (2013). Quality and structure of variability in children during motor development: A systematic review. Research in Developmental Disabilities, 34, 2810-2830. doi:10.1016/j. ridd.2013.05.031

Hamill, J., Palmer, C., \& Van Emmerik, R. E. A. (2012). Coordinative variability and overuse injury. Sports Medicine, Arthroscopy, Rehabilitation, Therapy \& Technology, 4, 45. doi:10.1186/1758-2555-4-45

Hausdorff, J. M., Edelberg, H. K., Mitchell, S. L., Goldberger, A. L., \& Wei, J. Y. (1997). Increased gait unsteadiness in community-dwelling elderly failers. Archives of Physical Medicine and Rehabilitation, 78, 278-283.

Jandová, S., Froňková, M., \& Nováková, K. (2013). Vliv obuvi nestabilní konstrukce na běžný krokový cyklus při chůzi na trenažeru při $10^{\circ}$ stoupání [Influence of instable construction shoes on usual gait cycle al walk on treadmill with $10^{\circ}$ ascent]. Rehabilitácia, 50, 226-231.

Kaipust, J. P., Huisinga, J. M., Filipi, M., \& Stergiou, N. (2012). Gait variability measures reveal differences between multiple sclerosis patients and healthy controls. Motor Control, 16, 229-244.

Miyoshi, H., Kinugasa, T., Urushihata, T., \& Soma, Y. (2011). Relationship between stride time variability of walking and fall experience in middle aged and elderly woman. Japanese Journal of Physical Fitness and Sports Medicine, 60, 121-132.

Perry, J., \& Burnfield, J. M. (2010). Gait analysis: Normal and pathological function (2nd ed.). Thorofare, NJ: SLACK Incorporated.

Peters, M. (1988). Footedness: Asymmetries in foot preference and skill and neuropsychological assessment of foot movement. Psychological Bulletin, 103, 179-192.

Reelick, M. F., Kessels, R. P. C., Faes, M. C., Weerdesteyn, V., Esselink, R. A. J., \& Olde Rikkert, M. G. M. (2011). Increased intra-individual variability in stride length and reaction time in recurrent older fallers. Aging Clinical and Experimental Research, 23, 393-399. doi:10.3275/7327

Taylor, M. E., Delbaere, K., Mikolaizak, A. S., Lord, S. R., \& Close, J. C. T. (2013). Gait parameter risk factors for falls under simple and dual task conditions in cognitively impaired older people. Gait \& Posture, 37, 126-130. doi:10.1016/j.gaitpost.2012.06.024

Wang, Y., \& Watanabe, K. (2012). Limb dominance related to the variability and symmetry of the vertical ground reaction force and center of pressure. Journal of Applied Biomechanics, 28, 473-478.

Webster, K. E., Merory, J. R., \& Wittwer, J. E. (2006). Gait variability in community dwelling adults with Alzheimer disease. Alzheimer Disease and Associated Disorders, 20, 37-40. doi:10.1097/01.wad.0000201849.75578.de 